\title{
Brane backreactions and the Fischler-Susskind mechanism in conformal field theory
}

\author{
Christoph A. Keller» \\ Institut für Theoretische Physik, ETH Zürich \\ CH-8093 Zürich, Switzerland
}

\begin{abstract}
The backreaction of D-branes on closed string moduli is studied in perturbed conformal field theory. To this end we analyse the divergences in the modular integral of the annulus diagram. By the Fischler-Susskind mechanism, these divergences lead to additional terms in the bulk renormalisation group equations. We derive explicit expressions for these backreaction terms, and follow the resulting renormalisation group flow in several examples, finding agreement with geometric expectations.
\end{abstract}

${ }^{*}$ E-mail: kellerc@itp.phys.ethz.ch 


\section{Introduction}

A lot of recent work in string theory has dealt with the question of moduli and moduli stabilisation in realistic compactifications. In such setups two kinds of moduli appear. Closed string moduli correspond to deformations of the bulk theory, i.e. in geometric language to deformations of the compactification manifold. Open string moduli on the other hand correspond to deformations of the branes of the configuration.

String compactification can also be considered in the framework of two dimensional conformal field theory. The compactification is then no longer given by a Calabi-Yau manifold, but by a worldsheet CFT of the correct central charge. The branes of such a configuration are described by conformal boundary conditions. Closed string moduli are given by exactly marginal bulk operators, open string moduli by exactly marginal boundary operators, and the theory is deformed by inserting such integrated operators in the correlators. Arguably the CFT point of view is more fundamental, as it includes all $\alpha^{\prime}$ corrections. On the other hand only for very few geometric configurations the corresponding worldsheet CFT is known explicitly.

To determine the moduli space of the theory, one needs to find all exactly marginal operators. A marginal operator is exactly marginal if it remains marginal in the perturbed theory, or, to put it another way, if it does not run under the renormalisation group flow. Criteria for this have been worked out for bulk [1] and boundary operators [2].

More recently, [3] considered the interplay between bulk and boundary operators. In particular, renormalisation group flow equations were derived which describe the effects of bulk perturbations on the boundary. These equations describe how the open string moduli space changes as bulk perturbations are turned on (see also [4 for a discussion of this question). More generally, they show that the boundary conditions flow to a fixed point which is compatible with the new, perturbed bulk theory. The bulk theory, however, remains fixed and is not affected by the boundary conditions - the brane does not backreact on the bulk.

The aim of this paper is to extend the RG equations of [3] to include the backreaction of branes on the bulk theory. The idea for the underlying mechanism goes back to [5, 6, 7, 8, 9]: in string theory, to calculate amplitudes one considers not only the disk diagram, but also diagrams of higher genus. The total amplitude is obtained by summing over all topologies and integrating over the moduli of the conformal structure of the diagrams. This integration can lead to new divergences at the boundary of the moduli space $\mathcal{M}$, i.e. when the surface degenerates. More precisely, the spectrum of the theory may contain tadpoles, i.e. massless modes, which give logarithmic divergences when integrated over $\mathcal{M}$. According to [7, 8], these can be absorbed by a suitable shift of the coupling constants in lower genus diagrams, thus contributing to the RG flow of the bulk couplings. Since the nature of the tadpoles depends on the boundary condition that is imposed, this describes the backreaction of the brane on the bulk.

We show that this prescription works for the annulus diagram, i.e. that the tadpole divergences can be compensated by local counterterms on the disk diagram, leading to additional terms in the bulk RG equations of [3]. The brane backreaction can thus be incorporated quite naturally in the language of renormalisation group flows.

The RG equations so obtained can be used to study various examples. In many cases, we know already from geometric considerations how the brane should deform the bulk 
theory, so that we can compare our results. For instance, we expect that a D1-brane wrapping a circle should shrink its radius. This is confirmed by the RG analysis. In other, more complicated examples we also find agreement between the RG analysis and geometric expectations or supergravity calculations.

This paper is organised as follows. In section 2 we first rederive the bulk-boundary RG equations of [3] using a different regularisation scheme which is more suitable for further analysis. We then derive the backreaction term to first order in the string coupling constant $g_{s}$ by analysing divergences of the annulus diagram. In section 3 we apply the extended RG equations to the free boson and WZW models. Section 4 discusses bosonic string theory in flat space and its relation to supergravity solutions. Finally, section 5 contains our conclusions.

\section{Renormalisation group equations}

\subsection{Dimensional regularisation on the disk}

Let us first derive the renormalisation group equations on the disk [10, 3]. Consider the partition function $\left\langle e^{-S}\right\rangle$, where $S$ is the perturbed action,

$$
S=S^{*}-\Delta S=S^{*}-\sum_{i} \lambda_{i} \ell^{h_{\phi_{i}}-2} \int \phi_{i}(z) d^{2} z-\sum_{j} \mu_{j} \ell^{h_{\psi_{j}}-1} \int \psi_{j}(x) d x
$$

We have introduced the length scale $\ell$ to keep the coupling constants dimensionless. Expanding $\left\langle e^{-S^{*}+\Delta S}\right\rangle$ in powers of $\lambda_{i}$ and $\mu_{j}$ gives terms of the form

$$
\begin{aligned}
\frac{\lambda_{1}^{l_{1}} \cdots \mu_{1}^{m_{1}} \cdots}{l_{1} ! \cdots m_{1} ! \cdots} \prod_{i} \ell^{\left(h_{\phi_{i}}-2\right) l_{i}} & \prod_{j} \ell^{\left(h_{\psi_{j}}-1\right) m_{j}} \\
& \times \int\left\langle\phi_{1}\left(z_{1}^{1}\right) \phi_{1}\left(z_{2}^{1}\right) \cdots \phi_{2}\left(z_{1}^{2}\right) \cdots \psi_{1}\left(x_{1}^{1}\right) \cdots\right\rangle \prod d^{2} z_{k}^{i} \prod d x_{k}^{j} .
\end{aligned}
$$

Here the bulk fields $\phi_{i}$ are integrated over the entire disk, and the boundary fields $\psi_{j}$ over its boundary. The disk has the conformal symmetry group $S U(1,1)$. The integration measure $d \mu$ must transform with conformal weight $(-1,-1)$ under such transformations, so that integrals of marginal $(1,1)$ fields $\int d \mu \phi_{(1,1)}$ are invariant. Clearly, $d^{2} z$ satisfies this property. Since we can use $S U(1,1)$ to map any point to 0 , it follows that up to a constant factor this is the only possible measure.

Because of the symmetry group, the integrals in (2.2) are infinite. To render them finite, we use $S U(1,1)$ to fix the position of one bulk and one boundary insertion. Alternatively, we can (formally) divide by the volume of $S U(1,1)$.

The terms (2.2) are still infinite, since the integrand diverges when fields come close together. More precisely, three different situations can cause divergences: when two bulk fields come close to each other, when two boundary fields come close to each other, or when a bulk field comes close to the boundary. These three situations will lead to the three different terms in the RG equations (2.7) and (2.8) below. We thus have to introduce a scheme to regularise the divergences. One such scheme [10, 3] is to cut out small disks of radius $a$ around all operators. 
Instead we will use a scheme which resembles dimensional regularisation. To evaluate diverging integrals, we change the conformal dimension of the fields involved to such values that the integral converges, and evaluate the original integral by analytic continuation. One motivation for using this scheme comes from the spacetime interpretation of the divergences that will show up in the modular integrals: they can be interpreted as infrared divergences due to massless modes, so that a natural regularisation is to introduce a small mass term. In the worldsheet theory, this corresponds to a shift of the conformal dimension of the field. From a more technical point of view, it is favourable to keep conformal covariance of all expressions, which is destroyed if we cut out small disks.

Let us shift the conformal weight of boundary fields as $h_{\psi} \mapsto h_{\psi}-\epsilon$, and that of bulk fields as $h_{\phi} \mapsto h_{\phi}-2 \epsilon \oplus^{\dagger}$ As an example for how the scheme works, consider two marginal bulk fields $\phi_{i}, \phi_{j}$ that come close to each other to produce another marginal field $\phi_{k}$,

$$
\lambda_{i} \ell^{-2 \epsilon} \lambda_{j} \ell^{-2 \epsilon} \phi_{i}(z) \phi_{j}(0) \sim \lambda_{i} \lambda_{j} \ell^{-4 \epsilon} \frac{\phi_{k}(0) C_{i j k}}{|z|^{h_{i}+h_{j}-h_{k}}}=\lambda_{i} \lambda_{j} \ell^{-4 \epsilon} \phi_{k}(0) C_{i j k}|z|^{-2+2 \epsilon}
$$

For simplicity, we have fixed the position of $\phi_{j}$ to 0 . We perform the $d^{2} z$ integral up to some IR cutoff $L$ to obtain

$$
\lambda_{i} \lambda_{j} \ell^{-2 \epsilon} \phi_{k}(0) 2 \pi C_{i j k} \frac{\ell^{-2 \epsilon}}{2 \epsilon} L^{2 \epsilon}
$$

We have pulled out a factor $\ell^{-2 \epsilon}$ which will be absorbed in the shift of $\lambda_{k}$ (see (2.6) $)$ ). The second factor $\ell^{-2 \epsilon}$ gives

$$
\frac{\ell^{-2 \epsilon}}{2 \epsilon} L^{2 \epsilon}=\frac{1}{2 \epsilon}-\log \ell+\log L+\mathcal{O}(\epsilon)
$$

In the limit $\epsilon \rightarrow \infty$, only the second term gives a dependence on $\ell$ which contributes to the $\mathrm{RG}$ flow. We see that the regularisation scheme has introduced an implicit dependence of the integral on $\ell$. As $\left\langle e^{-S}\right\rangle$ must be independent of $\ell$, we must compensate a shift in $\log \ell$ by shifting $\lambda_{i}$ and $\mu_{j}$. A combinatorial analysis shows that the shift needed is

$$
\lambda_{k} \ell^{-2 \epsilon} \mapsto \lambda_{k} \ell^{-2 \epsilon}+\lambda_{i} \lambda_{j} \ell^{-2 \epsilon} \pi C_{i j k} \cdot \log \ell .
$$

In a similar way, we treat the other types of divergences. The resulting renormalisation group equations are [3]

$$
\begin{aligned}
\dot{\lambda}_{k} & =\left(2-h_{\phi_{k}}\right) \lambda_{k}+\pi C_{i j k} \lambda_{i} \lambda_{j}+\mathcal{O}\left(\lambda^{3}\right), \\
\dot{\mu}_{k} & =\left(1-h_{\psi_{k}}\right) \mu_{k}+\frac{1}{2} B_{i k} \lambda_{i}+D_{i j k} \mu_{i} \mu_{j}+\mathcal{O}\left(\mu \lambda, \mu^{3}, \lambda^{2}\right),
\end{aligned}
$$

where the dot indicates a derivative with respect to the flow parameter $t=\log \ell$. To obtain higher order terms in $\mu$ and $\lambda$, one would have to analyse the situation when three or more fields come close to each other. In the following, we shall never consider such terms.

\footnotetext{
${ }^{\dagger}$ Note that for bulk fields in a theory with boundary $h=h_{L}+h_{R}$.
} 

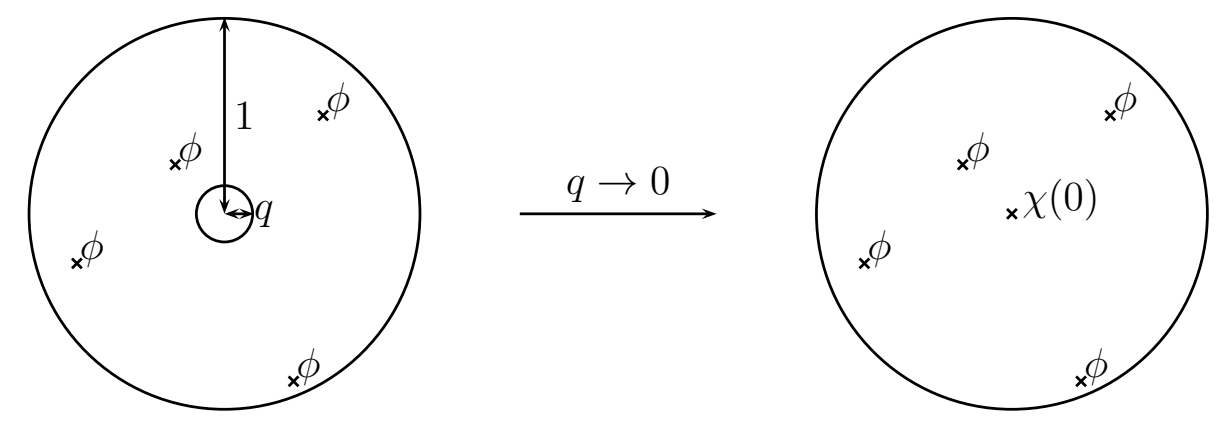

Figure 1: Divergences of the annulus diagram

\subsection{Higher genus: general strategy}

To calculate amplitudes in string theory, we have to take into account higher genus diagrams as well. For simplicity assume that there is only one type of field $\phi$ in our theory. As before, a string amplitude $F$ can be expanded in powers of $\lambda, F=\sum_{n} \lambda^{n} F_{n}$. Each term $F_{n}$ itself contains contributions from all topologically different diagrams with $n$ insertions of $\phi$. Moreover, for a given topology we must integrate over all conformal structures, parametrised by modular parameters $t_{i}$. In full,

$$
F_{n}=\sum_{k} g_{s}^{\chi_{k}} \int_{\mathcal{M}_{k}} d t_{i} F_{n}^{k}\left(t_{i}\right)
$$

where $g_{s}$ is the string coupling constant and $\chi_{k}$ is the Euler characteristic of the diagram $F^{k}$. Integration over the moduli space $\mathcal{M}_{k}$ leads to new divergences due to marginal and relevant modes in the spectrum of the theory. The divergences have to be regularised, and we must try to compensate for them by introducing counterterms on diagrams of lower genus. These $\ell$-dependent terms then give the the backreaction terms in the bulk $\mathrm{RG}$ equations.

\subsection{The annulus diagram}

We will now calculate the backreaction terms caused by the annulus diagram $A_{n}=F_{n}^{1}$. The annulus has a single real modular parameter $q$, its inner radius. The integral over $q$ produces a divergence for $q \rightarrow 0$. In this case there is an intuitive way to see how the counterterm on the disk arises, as shown in figure 1: the divergent part of the annulus diagram with $n$ integrated insertions corresponds to a disk diagram with an additional field $\chi(0)$ inserted. A shift $\lambda \mapsto \lambda+\delta \lambda$ on the disk diagram $D_{n+1}=F_{n+1}^{0}$ can thus compensate the divergence. The corresponding term is of order $g_{s}$.

Although we will only calculate the term of order $g_{s} \lambda^{0}$, some comments on terms of higher order in $\lambda$ are necessary. The analysis on the disk showed that $\lambda^{2}$ terms are produced by two fields approaching each other, and that higher order terms appear when $n$ fields come close together. In the situation here, higher order corrections arise when additional fields move close to the new field produced on the disk or to the boundary of the annulus. If 
for instance a single $\phi$ moves close to the centre of the annulus $A_{n}$, the divergence can be compensated by the disk diagram $D_{n}$, which produces a contribution of order $g_{s} \lambda$. As we are only interested in the lowest order correction, we can thus subtract divergences which arise from fields moving close to each other or to the boundary.

Note that the symmetry group of the annulus is only $U(1)$ - we can fix the position of one boundary insertion, or alternatively we can divide the amplitude by $2 \pi$. This also means that unlike on the disk, the conformal symmetry no longer uniquely fixes the integration measure. Nevertheless, the correct measure is still $d^{2} z$, see e.g. [11].

For a given radius $q$, the integrated $n$-point amplitude of the annulus is given by

$$
A_{n}(q)=\frac{1}{\pi} \prod_{i=1}^{n} \int_{1}^{q} d^{2} z_{i}\left\langle\left\langle B \| \phi\left(z_{1}\right) \ldots \phi\left(z_{n}\right) q^{L_{0}+\bar{L}_{0}-2}|| B\right\rangle\right\rangle .
$$

For simplicity, we have only included one type of marginal field $\phi$. As usual, $\langle\langle B \|$ is the boundary state at the outer radius 1 . To obtain the boundary state at the inner radius, we transport ||$B\rangle\rangle$ to the inner radius $q$ using the propagator $\pi^{-1} q^{L_{0}+\bar{L}_{0}-2}$, whose normalisation is fixed by the construction of the boundary states. By inserting a complete set of states, we expand the boundary state in a sum of fields inserted at the point 0 . The action of the propagator then gives

$$
\left.\left.\left.\pi^{-1} q^{L_{0}+\bar{L}_{0}-2}|| B\right\rangle\right\rangle=\pi^{-1} \sum_{i} q^{h_{i}+\bar{h}_{i}-2}\left|\phi_{i}\right\rangle\left\langle\phi_{i}|| B\right\rangle\right\rangle
$$

Here $\left.\left\langle\phi_{i}|| B\right\rangle\right\rangle$ is the disk one-point function with $\phi_{i}$ sitting at the point 0 . Integrating (2.10) over its moduli space using the measure $q^{-1} d q$, we see from (2.11) that divergences arise for $q \rightarrow 0$ for all fields with $h_{i}=\bar{h}_{i} \leq 1$. In a supersymmetric setup, we expect no relevant, i.e. tachyonic fields. In the bosonic theories we will consider, the only such field is usually the vacuum $h=\bar{h}=0$. The vacuum only changes overall normalisations, so that we will ignore it in what follows. The only divergences are then due to marginal fields $h_{i}=\bar{h}_{i}=1-\epsilon$. Their contribution is

$$
\left.\| B(q)\rangle\rangle \simeq \frac{q^{-2 \epsilon}}{\pi} \sum_{i}\left\langle\phi_{i}|| B\right\rangle\right\rangle \phi_{i}(0) .
$$

For the moment, let us assume that there are no integrated bulk insertions. The integral of (2.12) over moduli space converges if $\epsilon<0$, and we will use its analytic continuation,

$$
\left.\left.\left.\int_{0}^{1} q^{-1} d q \| B(q)\right\rangle\right\rangle=-\frac{1}{\pi} \frac{1}{2 \epsilon} \sum_{i}\left\langle\phi_{i}|| B\right\rangle\right\rangle \phi_{i}(0) .
$$

The pole in $\epsilon$ will then contribute to the RG equations as in (2.4).

If the diagram contains integrated bulk insertions, the comparison is a bit more subtle: in the disk diagram, the additional bulk insertions are integrated over the entire disk, whereas on the annulus they are only integrated up to the inner radius $q$. The divergent contribution of the tadpole, however, comes from the limit $q \rightarrow 0$. We can thus concentrate on annulus diagrams where $q<|\epsilon|$. Indeed,

$$
\int_{|\epsilon|}^{1} d q q^{-1-2 \epsilon}=-\frac{1}{2 \epsilon}\left(1-e^{-2 \epsilon \ln |\epsilon|}\right)=\mathcal{O}(\ln |\epsilon|)
$$


is only a subleading contribution compared to (2.13). We claim then that to lowest order in $\lambda$ we can rewrite the annular integral as

$$
\int_{0}^{|\epsilon|} d q \int_{q}^{1} d^{2} z_{i}\langle\ldots\rangle=\int_{|\epsilon|}^{1} d^{2} z_{i} \int_{0}^{|\epsilon|} d q\langle\ldots\rangle+\mathcal{O}\left(\epsilon^{2}\right)
$$

This holds because we can estimate the contribution of the fields $\phi$ integrated over the small disk of radius $|\epsilon|$ : since we only calculate the lowest order term in $\lambda$, we subtract all singular terms in $\phi$. The remaining expression is then bounded by some constant $B$, and we can estimate its contribution as $\leq \pi \epsilon^{2} B$. A similar argument shows that we can cut out the same small disk in the disk diagram without changing the result. This shows that we can compare annulus diagrams with disk diagrams even if they contain integrated insertions.

So far, the fields $\phi_{i}$ introduced by the tadpoles are inserted at the point $z=0$. In order to be able to compensate them with a disk diagram, we need to rewrite them as integrated insertions. To do this, we use the fact that the disk has a larger symmetry group than the annulus. Consider the disk diagram with $n$ integrated fields $\phi\left(z_{i}\right)$ and one additional field $\chi(z)$, each of them marginal. We can use part of the symmetry group $S U(1,1)$ to fix the position of $\chi$ to 0 . In particular, for each $z$ choose $f_{z} \in S U(1,1)$ such that $f_{z}(z)=0$. Defining $\hat{z}_{i}=f_{z}\left(z_{i}\right)$, conformal covariance tells us that the $z_{i}$ integral changes as

$$
\int d^{2} z_{i} \phi\left(z_{i}\right) \rightarrow \int d^{2} \hat{z}_{i}\left|\frac{\partial z_{i}}{\partial \hat{z}_{i}}\right|^{-2 \epsilon} \phi\left(\hat{z}_{i}\right)=\int d^{2} \hat{z}_{i} \phi\left(\hat{z}_{i}\right)+\mathcal{O}(\epsilon)
$$

Up to terms of order $\epsilon$, the resulting integral is thus independent of $z$, and the additional field $\chi(z)$ is fixed at the position $z=0$. Formally, we can write this manipulation as

$$
\frac{1}{|S U(1,1)|} \int d^{2} z \int d^{2} z_{i}\left\langle\chi(z) \phi\left(z_{1}\right) \ldots\right\rangle=\frac{1}{|U(1)|} \int d^{2} \hat{z}_{i}\left\langle\chi(0) \phi\left(\hat{z}_{1}\right) \ldots\right\rangle+\mathcal{O}(\epsilon),
$$

where $|G|$ denotes the volume of the respective symmetry group. On the right hand side of (2.17), we divide by $|U(1)|$ because we still have not fixed the entire symmetry: after choosing $f_{z}$, we can always rotate the disk around its centre. This remaining $U(1)$ symmetry however is exactly the symmetry group of the annulus, so that the right hand side of (2.17) is the standard annulus diagram with one fixed insertion.

The upshot of this analysis is that the divergent part of $A_{n}$ has the same form as $D_{n+1}$, so that it can be compensated by introducing a counterterm on the disk diagram. As before, we need to split off a factor $\ell^{-2 \epsilon}$ to be included in $\lambda$. The annulus contribution to the disk diagram is thus

$$
\left.\left.-\ell^{-2 \epsilon} \frac{g_{s}}{\pi} \frac{\ell^{2 \epsilon}}{2 \epsilon}\left\langle\phi_{i}|| B\right\rangle\right\rangle \int d^{2} z \phi_{i}(z)=-\ell^{-2 \epsilon} \frac{g_{s}}{\pi}\left(\frac{1}{2 \epsilon}+\log \ell+\mathcal{O}(\epsilon)\right)\left\langle\phi_{i}|| B\right\rangle\right\rangle \int d^{2} z \phi_{i}(z)
$$

for each marginal field $\phi_{i}$. The usual combinatorial analysis shows that this can be compensated by shifting the coupling constant $\lambda_{i}$.

Putting everything together we obtain the modified bulk RG equations

$$
\left.\dot{\lambda}_{k}=\left(2-h_{\phi_{k}}\right) \lambda_{k}+\frac{g_{s}}{\pi}\left\langle\phi_{k}|| B\right\rangle\right\rangle+\pi C_{i j k} \lambda_{i} \lambda_{j}+\mathcal{O}\left(g_{s} \lambda, \lambda^{3}, g_{s}^{2}\right) .
$$




\section{WZW models and the free boson}

We now apply equation (2.19) to some examples. First we consider the free boson compactified on a circle, subject to Neumann or Dirichlet boundary conditions. Then we turn to Wess-Zumino-Witten models based on compact Lie groups. These models and their boundary states are very well understood and can be interpreted geometrically. We can thus check RG flow results against geometric expectations.

\subsection{The free boson on a circle}

Let $X(z, \bar{z})$ be the free boson compactified on a circle of radius $R, X \sim X+2 \pi R$. Its action is given by

$$
S=\frac{1}{2 \pi} \int d^{2} z \partial X \bar{\partial} X
$$

Neumann and Dirichlet boundary conditions are given by identifying on the real axis $z=\bar{z}$

$$
\partial X=\bar{\partial} X \text { (Neumann) } \quad \text { and } \quad \partial X=-\bar{\partial} X \text { (Dirichlet) }
$$

As usual, we can switch to the closed string picture by mapping the upper half-plane to the disk. The boundary condition is then described by the boundary states ||$N\rangle\rangle$ and $\| D\rangle\rangle$, respectively.

The ground states of the system are parametrised by momentum and winding numbers $n, w \in \mathbb{Z}$ such that

$$
\left(p_{L}, p_{R}\right)=\left(\frac{n}{2 R}+w R, \frac{n}{2 R}-w R\right),
$$

with conformal weight given by $\left(\frac{1}{2} p_{L}^{2}, \frac{1}{2} p_{R}^{2}\right)$. At a generic radius $R$, the only marginal operator is $\partial X \bar{\partial} X$. Its one-point function is given by

$$
\langle\partial X \bar{\partial} X|| N\rangle\rangle=1 \quad \text { and } \quad\langle\partial X \bar{\partial} X \| D\rangle\rangle=-1 .
$$

We will also have to deal with the relevant fields that are present in theory.

Let us analyse the Neumann case first. The one-point function vanishes unless $p_{L}=-p_{R}$, i.e. $n=0$, so that only pure winding modes couple. If we take $R$ big enough, (3.2) shows that all these modes become irrelevant. It is thus sufficient to only consider the perturbation by $\partial X \bar{\partial} X$,

$$
S=\frac{1}{2 \pi} \int d^{2} z \partial X \bar{\partial} X-\lambda \int d^{2} z \partial X \bar{\partial} X
$$

We see that (2.19) yields $\dot{\lambda}=g_{s} / \pi>0$. An increase in $\lambda$ means that the circle shrinks, as can be seen from (3.4): to maintain the correct normalisation of the action, we have to introduce rescaled fields $X^{\prime}=\sqrt{1-2 \pi \lambda} X$, which satisfy $X^{\prime} \sim X^{\prime}+2 \pi R^{\prime}=X^{\prime}+$ $2 \pi R \sqrt{1-2 \pi \lambda}$.

This shows that a Neumann brane that wraps the circle shrinks its radius. Similar reasoning shows that the D0 brane given by $\| D\rangle\rangle$ increases the radius of the circle.

When $R$ becomes of the order of the self-dual radius $R_{0}=1 / \sqrt{2}$, new relevant and marginal fields appear, and the above analysis breaks down. To analyse this case, we will use the fact that the free boson at the self-dual radius is equivalent to the $S U(2)$ Wess-Zumino-Witten-model at level 1. We therefore turn our attention to WZW-models. 


\subsection{Renormalisation group flows in general WZW models}

Wess-Zumino-Witten models are often described as $\sigma$-models on a group manifold of a Lie group $G$ [12]. A different, more algebraic approach is to define them via their operator content and correlation functions. For the moment, we will use this more abstract formulation, before changing to a more geometric picture in the next section.

The currents of the WZW model of a Lie group $G$ at level $k$ correspond to elements of the Lie algebra $\mathfrak{g}$ of $G$ and satisfy the operator product expansion

$$
J^{a}(z) J^{b}(w) \sim \frac{k \delta^{a b}}{(z-w)^{2}}+i f^{a b} \frac{J^{c}(w)}{(z-w)},
$$

where $f^{a b}{ }_{c}$ are the structure constants of $\mathfrak{g}$. The marginal fields of the theory are given by all possible combinations $J^{a} \bar{J}^{b}$ of left-moving and right-moving currents. We consider branes that preserve the affine symmetry up to conjugation by $g \in G$ [13, 14, 15]. In the closed string picture this means that the boundary state $\| B\rangle\rangle$ has to satisfy the gluing condition

$$
\left.\left.\left(g J_{m}^{a} g^{-1}+\bar{J}_{-m}^{a}\right)|| B\right\rangle\right\rangle=0
$$

whereas in the open string picture the left and right moving currents are identified at the boundary as

$$
g J^{a}(z) g^{-1}=\bar{J}^{a}(\bar{z}) \quad \text { for } z=\bar{z} .
$$

The one-point function is best evaluated in the open string picture and gives [16, 17]

$$
\left\langle\left(J^{a} \bar{J}^{b}\right)(u)\right\rangle_{B}=k \frac{\operatorname{tr}\left(J^{a} g J^{b} g^{-1}\right)}{(u-\bar{u})^{2}}=-k \frac{\operatorname{tr}\left(J^{a} g J^{b} g^{-1}\right)}{|u-\bar{u}|^{2}},
$$

so that $\left.\left\langle J^{a} \bar{J}^{b}|| B\right\rangle\right\rangle=-k \operatorname{tr}\left(J^{a} g J^{b} g^{-1}\right)$. Note that the currents are normalised such that $\operatorname{tr}\left(J^{a} J^{b}\right)=\delta^{a b}$. The orthonormal marginal fields are thus

$$
\phi_{a b}(z)=k^{-1} J^{a} \bar{J}^{b}
$$

Let us start from the model which is initially unperturbed. To lowest order, (2.19) gives then

$$
\dot{\lambda}_{a b}=-\frac{g_{s}}{\pi} \operatorname{tr}\left(J^{a} g J^{b} g^{-1}\right) .
$$

Higher order contributions in the bulk come from evaluating connected $n$-point functions. They are given [16, 17] by the product of traces $k \operatorname{tr}\left(J^{a_{1}} \ldots J^{a_{n}}\right) k \operatorname{tr}\left(\bar{J}^{b_{1}} \ldots \bar{J}^{b_{n}}\right)$, so that in the normalisation (3.9) they go as $k^{2-n}$. In the limit $k \rightarrow \infty$ they only give subleading contributions.

Let us make a side remark. We can choose an orthogonal basis $J^{a}, a=1, \ldots, r$ for the left moving currents, and a corresponding basis $\bar{J}^{b}:=g^{-1} J^{b} g, b=1, \ldots, r$ for the right moving currents. (3.10) then shows that only the fields $\phi_{a a}$ are switched on. Note that these fields leave the boundary conditions unchanged, as

$$
\left[t^{a}, g \bar{t}^{a} g^{-1}\right]=\left[t^{a}, t^{a}\right]=0
$$

which means that all $B_{i k}$ in (2.8) vanish, so that no boundary fields are switched on [3]. The brane changes the bulk without inducing a backreaction on itself. 


\subsection{Geometric interpretation of $S U(2)_{k}$}

To get a geometric picture of the brane backreaction, we switch to a more geometric description of WZW models. We will concentrate on $G=S U(2)$. We can write this theory as a $\sigma$-model on the group manifold, using the parametrisation [18]

$$
g=e^{i(\theta+\tilde{\theta}) \sigma_{2} / 2} e^{i \phi \sigma_{1} / 2} e^{-i(\theta-\tilde{\theta}) \sigma_{2} / 2},
$$

or explicitly

$$
g=\left(\begin{array}{cc}
\cos \frac{\phi}{2} \cos \tilde{\theta}+i \sin \frac{\phi}{2} \sin \theta & \cos \frac{\phi}{2} \sin \tilde{\theta}+i \sin \frac{\phi}{2} \cos \theta \\
-\cos \frac{\phi}{2} \sin \tilde{\theta}+i \sin \frac{\phi}{2} \cos \theta & \cos \frac{\phi}{2} \cos \tilde{\theta}-i \sin \frac{\phi}{2} \sin \theta
\end{array}\right) .
$$

At level $k$ the action then becomes

$$
S_{0}(\phi, \theta, \tilde{\theta})=\frac{k}{2 \pi} \int d^{2} z\left(\frac{1}{4} \bar{\partial} \phi \partial \phi+\sin ^{2} \frac{\phi}{2} \bar{\partial} \theta \partial \theta+\cos ^{2} \frac{\phi}{2} \bar{\partial} \tilde{\theta} \partial \tilde{\theta}+\cos ^{2} \frac{\phi}{2}(\bar{\partial} \theta \partial \tilde{\theta}-\bar{\partial} \tilde{\theta} \partial \theta)\right) \text {. }
$$

For later use, we also derive explicit expressions for the currents $J=-k \partial g g^{-1}$ and $\bar{J}=k g^{-1} \bar{\partial} g$,

$$
\begin{aligned}
J^{1} & =-k \frac{i}{\sqrt{2}}(\partial \phi \cos (\tilde{\theta}+\theta)-\partial \theta \sin \phi \sin (\tilde{\theta}+\theta)+\partial \tilde{\theta} \sin \phi \sin (\tilde{\theta}+\theta) \\
J^{2} & =-k \frac{i}{\sqrt{2}}(\partial \theta(1-\cos \phi)+\partial \tilde{\theta}(1+\cos \phi)) \\
J^{3} & =-k \frac{i}{\sqrt{2}}(\partial \phi \sin (\tilde{\theta}+\theta)+\partial \theta \sin \phi \cos (\tilde{\theta}+\theta)-\partial \tilde{\theta} \sin \phi \cos (\tilde{\theta}+\theta)
\end{aligned}
$$

and

$$
\begin{aligned}
\bar{J}^{1} & =k \frac{i}{\sqrt{2}}(\bar{\partial} \phi \cos (\tilde{\theta}-\theta)+\bar{\partial} \theta \sin \phi \sin (\tilde{\theta}-\theta)+\bar{\partial} \tilde{\theta} \sin \phi \sin (\tilde{\theta}-\theta)) \\
\bar{J}^{2} & =k \frac{i}{\sqrt{2}}(\bar{\partial} \theta(-1+\cos \phi)+\bar{\partial} \tilde{\theta}(1+\cos \phi)) \\
\bar{J}^{3} & =k \frac{i}{\sqrt{2}}(-\bar{\partial} \phi \sin (\tilde{\theta}-\theta)+\bar{\partial} \theta \sin \phi \cos (\tilde{\theta}-\theta)+\bar{\partial} \tilde{\theta} \sin \phi \cos (\tilde{\theta}-\theta)) .
\end{aligned}
$$

The boundary states are given by $\| j, g\rangle\rangle$. For each gluing condition $g$ there are $k+1$ possible branes, labelled by $j=0, \frac{1}{2}, \ldots, \frac{k}{2}$. [19] gives a geometric interpretation for these branes in terms of conjugacy classes: if $g$ is the identity $e$, then ||$j, e\rangle\rangle$ is the $S^{2}$ that wraps the conjugacy class given by

$$
h\left(\begin{array}{cc}
e^{2 \pi i j / k} & 0 \\
0 & e^{-2 \pi i j / k}
\end{array}\right) h^{-1} .
$$

In particular, for $j=0$ and $j=\frac{k}{2}$, the conjugacy class collapses to a point and the brane describes a D0 brane sitting at the point $e$ and $-e$, respectively. If the gluing map is given by a general $g$, the position of the brane shifts accordingly.

To go to the geometric limit, we fix $j$ and let $k \rightarrow \infty$. Independent of $j$ the brane thus becomes a D0 brane sitting at the point $g$. Also, (3.10) shows that the flow induced 
depends only on $g$. We can therefore suppress the index $j$ and parametrise the brane only by $g=g(\Phi, \Theta, \tilde{\Theta})$. Note that we denote its position by capital letters $\Phi, \Theta, \tilde{\Theta}$, as opposed to small letters for the coordinates of the manifold.

In the geometric limit the $S U(2)_{k}$ model corresponds to a non-linear $\sigma$-model on $S^{3}$ with radius $r \sim \sqrt{k}$. We can read off the target space metric $G$ and the field $B$ from the coefficients of the action. In the unperturbed case (3.14) this gives

$$
G_{0}=\left(\begin{array}{ccc}
k / 4 & 0 & 0 \\
0 & k \sin ^{2} \frac{\phi}{2} & 0 \\
0 & 0 & k \cos ^{2} \frac{\phi}{2}
\end{array}\right), \quad B_{0}=\left(\begin{array}{ccc}
0 & 0 & 0 \\
0 & 0 & k \cos ^{2} \frac{\phi}{2} \\
0 & -k \cos ^{2} \frac{\phi}{2} & 0
\end{array}\right) .
$$

\subsection{Minimising the brane mass}

Let us now calculate the RG flow and try to interpret it. (3.10) shows that the marginal fields $J^{i} \bar{J}^{j}$ are turned on with the respective strength

$$
\dot{\lambda}_{i j}(\Phi, \Theta, \tilde{\Theta})=-\frac{g_{s}}{\pi} \operatorname{tr}\left(J^{i} g J^{j} g^{-1}\right)=:-\frac{g_{s}}{\pi} K_{i j}(\Phi, \Theta, \tilde{\Theta}) .
$$

The coefficients $K_{i j}$ depend on the position of the brane and are given by

$K_{i j}=2\left(\begin{array}{ccc}\cos 2 \tilde{\Theta} \cos ^{2} \frac{\Phi}{2}+\cos 2 \Theta \sin ^{2} \frac{\Phi}{2} & \sin (\Theta+\tilde{\Theta}) \sin \Phi & \sin 2 \Theta \sin ^{2} \frac{\Phi}{2}-\sin 2 \tilde{\Theta} \cos ^{2} \frac{\Phi}{2} \\ -\sin (\Theta-\tilde{\Theta}) \sin \Phi & \cos \Phi & \cos (\Theta-\tilde{\Theta}) \sin \Phi \\ \sin 2 \Theta \sin ^{2} \frac{\Phi}{2}+\sin 2 \tilde{\Theta} \cos ^{2} \frac{\Phi}{2} & -\cos (\Theta+\tilde{\Theta}) \sin \Phi & \cos 2 \tilde{\Theta} \cos ^{2} \frac{\Phi}{2}-\cos 2 \Theta \sin ^{2} \frac{\Phi}{2}\end{array}\right)$.

This flow has a nice geometric interpretation. The mass of a brane is given by the value of the dilaton $\varphi$. Perturbing the metric of $S^{3}$ induces a non-constant dilaton and so changes the mass of the brane. [3] showed that in the case of an induced boundary flow, the brane deformed in such a way as to minimise its mass. We will show that a similar thing happens here: this time, the brane remains at the same place, but it deforms the geometry in such a way that its mass is minimised.

To show this, let us first find the change in geometry that decreases the mass of the brane as much as possible. The most general current-current deformation of the original theory is

$$
S=S_{0}-\alpha \int d^{2} z \sum_{i, j} a_{i j} J^{i}(z) \bar{J}^{j}(\bar{z})
$$

where the $a_{i j}$ are real coefficients. This gives a new metric $G^{\prime}(\phi, \theta, \tilde{\theta})=G_{0}-\alpha G_{1}$ and a new $B$-field. The new, nontrivial dilaton $\varphi$ can be calculated by [18, 20]

$$
e^{-2 \varphi_{0}} \sqrt{\operatorname{det} G_{0}}=e^{-2 \varphi(\phi, \theta, \tilde{\theta})} \sqrt{\operatorname{det} G^{\prime}(\phi, \theta, \tilde{\theta})} .
$$

The mass of the brane at $g=g(\Phi, \Theta, \tilde{\Theta})$ is proportional to $g_{s}^{-1} \sim e^{-\varphi(\Phi, \Theta, \tilde{\Theta})}$. We thus want to maximise the increase of $\operatorname{det} G_{0}^{-1} G^{\prime}$ at the point $(\Phi, \Theta, \tilde{\Theta})$. Its derivative is given by

$$
\left.\partial_{\alpha} \operatorname{det}\left(1-\alpha G_{0}^{-1} G_{1}\right)\right|_{0}=-\operatorname{tr} G_{0}^{-1} G_{1} .
$$

A straightforward calculation then shows

$$
\operatorname{tr} G_{0}^{-1} G_{1}(\Phi, \Theta, \tilde{\Theta})=k \sum_{i, j} a_{i j} K_{i j}(\Phi, \Theta, \tilde{\Theta})
$$


where $K_{i j}$ is the same expression as in (3.18). Introducing a Lagrange multiplier term $\nu \sum_{i, j} a_{i j}^{2}$ shows that the expression is extremised by $a_{i j}=-K_{i j}(\Phi, \Theta, \tilde{\Theta})$. Comparing to (3.17) we find perfect agreement.

We can try to follow the flow further and describe the geometry of the deformed manifold. By the symmetry of the problem, it is sufficient to consider the brane sitting at $\theta=0, \tilde{\theta}=$ dif so that

$$
g=\left(\begin{array}{cc}
e^{i \Phi / 2} & 0 \\
0 & e^{-i \Phi / 2}
\end{array}\right)
$$

(3.18) then turns on the fields

$$
\lambda_{i j}=-2 \frac{g_{s}}{\pi}\left(\begin{array}{ccc}
1 & 0 & 0 \\
0 & \cos \Phi & \sin \Phi \\
0 & -\sin \Phi & \cos \Phi
\end{array}\right)
$$

They change the metric $G_{0}$ by some expression $2 \frac{g_{s}}{\pi} G_{1}^{\Phi}(\phi, \theta, \tilde{\theta})$. At the point of the brane, $G_{1}^{\Phi}$ simplifies:

$$
G_{1}^{\Phi}(\Phi, 0,0)=\left(\begin{array}{ccc}
k^{2} / 2 & 0 & 0 \\
0 & 2 k^{2} \sin ^{2} \frac{\Phi}{2} & 0 \\
0 & 0 & 2 k^{2} \cos ^{2} \frac{\Phi}{2}
\end{array}\right)=2 k G_{0}(\Phi, 0,0)
$$

The effect of the backreaction is simply to rescale the original metric. We can continue to use our original reasoning even away from the point $t=0$ to obtain the differential equation

$$
\dot{G}^{\Phi}(\Phi, 0,0) \sim G_{0}(\Phi, 0,0) .
$$

The geometric analysis only gives the direction of the flow, so that we are free to choose the actual flow parameter. Writing

$$
G_{\mu \nu}^{\Phi}(t)=G_{0 \mu \nu}+4 \frac{g_{s}}{\pi} k t G_{1 \mu \nu}^{\Phi}
$$

we fix $t$ so that it agrees with the conformal field theory flow parameter at $t=0$.

Note that this analysis agrees with the observation in section 3.2, where we argued that in the limit $k \rightarrow \infty$, only the zero order term is important, and that thus no new bulk fields are turned on. This translates to the statement that (3.26) remains valid away from the starting point.

We can now try to understand how the geometry of the $S^{3}$ changes as we start to flow, and we can also try to estimate how far we should trust our analysis. Define a new flow parameter $t^{\prime}=4 g_{s} t / \pi$. Take the metric $G_{\mu \nu}^{\Phi}\left(t^{\prime}\right)$ and calculate the associated Ricci scalar $R\left(t^{\prime}\right)$. At the point $g$ it is given by

$$
R\left(t^{\prime}\right)=\frac{6+84 k t^{\prime}}{k\left(1+2 k t^{\prime}\right)^{2}}
$$

The curvature thus increases at first, in agreement with the intuition that the brane warps the space around it.

\footnotetext{
${ }^{\ddagger}$ We could simply restrict to $g=e$, but $e$ is a coordinate singularity in the parametrisation chosen.
} 
The geometric picture breaks down as soon as the curvature becomes too big. In fact, if one considers $R\left(t^{\prime}\right)$ on all of $S^{3}$, it turns out that at $k t^{\prime}=\frac{1}{2}$ the curvature becomes singular at some points. The geometric approximation thus becomes unreliable as soon as $k t^{\prime} \sim 1$. In particular, one should not trust (3.28) for values $k t^{\prime} \sim \frac{5}{14}$, where $R\left(t^{\prime}\right)$ seemingly starts to decrease.

\section{Flat space}

The last example we consider is the bosonic string in flat space in the presence of a $\mathrm{D} p$ brane. In this case, one can consider the low-energy supergravity limit of the theory. The D-brane is then given by a $p$-brane, a solution of the corresponding supergravity equation. [21] performed a boundary state calculation and found agreement with the supergravity results. We will reproduce their results using the extended RG equations.

\subsection{The boundary state}

The conformal field theory is described by 26 free bosons with ladder operators $a_{n}^{\mu}, \bar{a}_{n}^{\nu}$. A $\mathrm{D} p$-brane located at $y$ is described by the boundary state [21]

$$
\| D p ; y\rangle\rangle=\frac{T_{p}}{2} \int \frac{d^{d_{\perp}} k_{\perp}}{(2 \pi)^{d_{\perp}}} e^{i k_{\perp} y} \exp \left[-\sum_{n=1}^{\infty} a_{-n}^{\mu} \mathcal{S}_{\mu \nu} \bar{a}_{-n}^{\nu}\right]\left|0 ; k_{\|}=0, k_{\perp}\right\rangle .
$$

The diagonal matrix $\mathcal{S}_{\mu \nu}$ is given by

$$
\mathcal{S}_{\mu \nu}=\left(\eta_{\alpha \beta},-\delta_{i j}\right)
$$

where $\alpha, \beta$ run over the $d_{\|}=p+1$ dimensions parallel to the brane, and $i, j$ over the $d_{\perp}=26-p-1$ transverse dimension. Its tension is

$$
T_{p}=\frac{\sqrt{\pi}}{2^{(d-10) / 4}}\left(4 \pi^{2} \alpha^{\prime}\right)^{(d-2 p-4) / 4} .
$$

Again, we will ignore the tachyon and concentrate on marginal fields. The corresponding states are of the form

$$
a_{-1}^{\mu} \bar{a}_{-1}^{\nu}|0 ; k\rangle \text {. }
$$

Here $|0 ; k\rangle$ is the ground state of momentum $k$, normalised as $\left\langle k \mid k^{\prime}\right\rangle=2 \pi \delta\left(k-k^{\prime}\right)$, with $(2 \pi)^{d} \delta^{(d)}(0)=V$. The conformal weight of (4.4) is $\left(1+\alpha^{\prime} k^{2} / 4,1+\alpha^{\prime} k^{2} / 4\right)$, and it couples to the $\mathrm{D} p$-brane centred at $y=0$ as

$$
\left.A_{k}^{\mu \nu}:=\left\langle 0 ; k \mid a_{1}^{\mu} \bar{a}_{1}^{\nu} \| D p ; 0\right\rangle\right\rangle=-\frac{T_{p}}{2} \delta^{\left(d_{\|}\right)}\left(k_{\|}\right) \mathcal{S}^{\mu \nu} .
$$

We see that only states with $k_{\|}=0$ couple to the brane. It is thus necessary to consider states with non-vanishing transverse momentum, which means $k_{\perp}^{2}>0$, such that $k^{2}>0$. This poses a problem, as in string theory vertex operators have to be marginal, so that $k^{2}=0$.

This analysis indicates that we need to go off-shell to find states that couple to the brane. From the CFT point of view such this means that we need to consider states (4.4) that are almost marginal. 


\subsection{Applying the RG equations}

We would like to apply (2.19) and find the fixed point to which the theory flows. Although we derived (2.19) only for marginal fields, the argument also works for almost marginal fields with $h=1+\delta h$. $\delta h$ then takes the role of $\epsilon$, and the counterterm needed is $\sim \ell^{\delta h}(\delta h)^{-1}$. The contribution to (2.19) is again $\left.\frac{g_{s}}{\pi}\left\langle\phi_{k}|| B\right\rangle\right\rangle$. It is clear however that several steps of the derivation depended on taking $\epsilon \rightarrow 0$ in the end. We should therefore trust (2.19) only for almost marginal fields with $\delta h \ll 1$.

A fixed point of (2.19) is given by

$$
0=\dot{\lambda}^{\mu \nu}=(2-h) \lambda^{\mu \nu}+\frac{g_{s}}{\pi} A_{k}^{\mu \nu}+\mathcal{O}\left(g_{s} \lambda, \lambda^{3}, g_{s}^{2}\right)
$$

so that to lowest order

$$
\lambda^{\mu \nu}=\frac{2 g_{s}}{\pi \alpha^{\prime}} \frac{A_{k}^{\mu \nu}}{k_{\perp}^{2}}=\frac{g_{s} T_{p}(2 \pi)^{p+1} V_{p+1}}{\pi \alpha^{\prime}} \frac{\mathcal{S}^{\mu \nu}}{k_{\perp}^{2}} .
$$

To compare to the metric in the supergravity solution, we calculate the expectation value of the graviton, i.e. its one-point function. Assuming that the fields $\phi_{\mu \nu}(k)$ were orthonormal in the original theory, the perturbed one-point function of $a_{-1}^{\mu} \bar{a}_{-1}^{\nu}|0 ; k\rangle$ is

$$
\left\langle\phi^{\mu \nu}(k)\right\rangle_{\lambda}=\lambda^{\sigma \rho}\left\langle\phi_{\sigma \rho}(k) \phi^{\mu \nu}(k)\right\rangle_{0}+O\left(\lambda^{2}\right)=\lambda^{\mu \nu}+O\left(\lambda^{2}\right) .
$$

To obtain the expectation value of the graviton, we have to extract the symmetric traceless part of (4.7), as has been done in [21]. (4.7) then agrees with their results, up to a constant factor due to different normalisations.

Our analysis is only valid if $\alpha^{\prime} k_{\perp}^{2} \ll 1$, since else $\delta h$ is too big. Moreover $\frac{g_{s} T_{p}}{\alpha^{\prime} k_{\perp}^{2}} \ll 1$ is needed, since otherwise higher order terms will become important. Geometrically this means that we can only consider weakly curved configurations, and only probe the long distance limit. Our analysis is thus valid in the same range of parameters as the supergravity calculation.

\section{Conclusions}

We have calculated the backreaction of a brane on the bulk theory. The RG equations so obtained are a concrete realisation of the Fischler-Susskind mechanism. For the free boson on a circle and for the $S U(2)$ WZW-model, the resulting flows agree with geometric expectations. For flat space, we are able to reproduce the long-distance behaviour of the supergravity solution.

An obvious extension of this work is to try to include higher order terms in $g_{s}$. Technically, this is probably quite challenging. There is however a more fundamental question: the analysis of section 2 shows that annulus tadpoles can be compensated by local counterterms, i.e. that their effect can be expressed by standard RG equations. It is not clear that this will also work for higher order tadpoles, e.g. for the disk with one thin handle that shrinks to zero thickness.

The other natural extension is to generalise the RG equations to theories with worldsheet supersymmetry. This would allow to consider setups that are phenomenologically more 
interesting. In particular, one could investigate supersymmetric configurations similar to [22]. In such a setup, shifting the closed string moduli away from their original, supersymmetric values will induce a flow in the configuration of branes. These in turn will backreact on the bulk. It would be interesting to find the end point of this combined flow and to check if the resulting theory is again supersymmetric.

\section{Acknowledgements}

I want to thank my advisor Matthias Gaberdiel for suggesting this topic to me, and for his constant support and advice during the project itself. I would also like to thank Costas Bachas, Ben Craps, Stefan Fredenhagen, Ingo Kirsch, and Stefan Stieberger for helpful discussions.

\section{References}

[1] S. Chaudhuri and J.A. Schwartz, A criterion for integrably marginal operators, Phys. Lett. B 219 (1989) 291.

[2] A. Recknagel and V. Schomerus, Boundary deformation theory and moduli spaces of D-branes, Nucl. Phys. B 545 (1999) 233 [arXiv:hep-th/9811237].

[3] S. Fredenhagen, M.R. Gaberdiel and C.A. Keller, Bulk induced boundary perturbations, J. Phys. A 40 (2007) F17 [arXiv:hep-th/0609034].

[4] S. Fredenhagen, M.R. Gaberdiel and C.A. Keller, Symmetries of perturbed conformal field theories, arXiv:0707.2511 [hep-th].

[5] E. Cremmer and J. Scherk, Factorization of the pomeron sector and currents in the dual resonance model, Nucl. Phys. B 50 (1972) 222.

[6] L. Clavelli and J.A. Shapiro, Pomeron factorization in general dual models, Nucl. Phys. B 57 (1973) 490.

[7] W. Fischler and L. Susskind, Dilaton tadpoles, string condensates and scale invariance, Phys. Lett. B 171 (1986) 383.

[8] W. Fischler and L. Susskind, Dilaton tadpoles, string condensates and scale invariance. 2, Phys. Lett. B 173 (1986) 262.

[9] C.G. Callan, C. Lovelace, C.R. Nappi and S.A. Yost, String loop corrections to beta functions, Nucl. Phys. B 288 (1987) 525.

[10] J.L. Cardy, Conformal invariance and statistical mechanics, in: 'Fields, strings and critical phenomena', proceedings of the Les Houches summer school 1988, North Holland (1990).

[11] M.B. Green, J.H. Schwarz and E. Witten, Superstring Theory. Vol. 2: Loop amplitudes, anomalies and phenomenology, CUP. (1987). 
[12] E. Witten, Nonabelian bosonization in two dimensions, Commun. Math. Phys. 92 (1984) 455.

[13] M.R. Gaberdiel, A. Recknagel and G.M.T. Watts, The conformal boundary states for SU(2) at level 1, Nucl. Phys. B 626 (2002) 344 [arXiv:hep-th/0108102].

[14] C.G. Callan, I.R. Klebanov, A.W.W. Ludwig and J.M. Maldacena, Exact solution of a boundary conformal field theory, Nucl. Phys. B 422 (1994) 417 [arXiv: hep-th/9402113].

[15] J. Polchinski and L. Thorlacius, Free fermion representation of a boundary conformal field theory, Phys. Rev. D 50 (1994) 622 [arXiv:hep-th/9404008].

[16] I.B. Frenkel and Y. Zhu, Vertex operator algebras associated to representations of affine and Virasoro algebras, Duke Math. J. 66 (1992) 123.

[17] M.R. Gaberdiel and P. Goddard, Axiomatic conformal field theory, Commun. Math. Phys. 209 (2000) 549 [arXiv:hep-th/9810019].

[18] S.F. Hassan and A. Sen, Marginal deformations of WZNW and coset models from $O(D, D)$ transformation, Nucl. Phys. B 405 (1993) 143 [arXiv:hep-th/9210121].

[19] A.Y. Alekseev and V. Schomerus, D-branes in the WZW model, Phys. Rev. D 60 (1999) 061901 [arXiv:hep-th/9812193].

[20] S. Förste, D-branes on a deformation of SU(2), JHEP 0202 (2002) 022 [arXiv: hep-th/0112193].

[21] P. Di Vecchia, M. Frau, I. Pesando, S. Sciuto, A. Lerda and R. Russo, Classical pbranes from boundary state, Nucl. Phys. B 507 (1997) 259 [arXiv:hep-th/9707068].

[22] M. Cvetic, G. Shiu and A.M. Uranga, Chiral four-dimensional $N=1$ supersymmetric type IIA orientifolds from intersecting D6-branes, Nucl. Phys. B 615 (2001) 3 [arXiv:hep-th/0107166]. 